\title{
ZU EINIGEN MITTELALTERLICHEN AMTSBEZEICHUNGEN IN DER TOPONYMIE SERBIENS
}

Der vorliegende Aufsatz knüpft an meine jahrelange Beschäftigung mit der um 1316 abgefassten Gründungsurkunde des Klosters Banjska an, woraus sich vor kurzem ein Buch ergeben hat (Loma 2013). Ebendort S. 231 wird der Ortsname (ON) Čigotovo (heute Čikatovo) im Kosovogebiet als Possessivbildung zum Titel čigot erklärt, der bislang nur aus dem kirchenslavischen Übersetzungsschrifttum bekannt war, wо чиготъ gr. ( $\pi \rho \omega \tau 0) \sigma \pi \alpha \theta$ áptos (wörtlich: 'Schwertträger', daraus 'Leibwächter', vom 9. bis zum 11. Jh. ein byzantinischer Hoftitel) wiedergibt. ${ }^{1}$ Inzwischen steht fest, dass dieser Titel auf altserbischem Boden gebräuchlich war. Es ist sogar möglich, die urkundliche Angabe so zu interpretieren, dass zu jener Zeit ein gewisser Dobroslav Grbica das Amt des čigot am Hof des serbischen Königs Milutin bekleidete. ${ }^{2}$ Eine weitere toponymische Spur dürfte das Wort in Kroatien hinterlassen haben, wo der ON Čigoč unweit von Sisak ein $j$-Possessivum davon zu sein scheint. Damit erlangte eine hochsprachliche Amtsbezeichnung, wohl orientalischer Herkunft, ${ }^{3}$ ihre historisch-geographische Verankerung. Im Folgenden wird ein ähnlicher Fall aus der Toponymie Serbiens erörtert.

Südöstlich von Belgrad, unweit der Kleinstadt Mladenovac, am Bach Ralja im Einzugsgebiet der Großen Morava, liegt das Dorf Šepšin. Unter dem heutigen Namen wird es erstmals 1818 als zur damaligen Nahija von Grocka gehörig erwähnt (Vuk 1827: 69). Aus türkischen Quellen erfahren wir, dass es im 16. Jh. in derselben Gegend zwei benachbarte Dörfer mit ähnlich klingenden Namen gab. Bei diesen Quellen handelt es sich um die türkischen Steuerverzeichnisse (Defter) Belgrads und seiner Umgebung, die bald nach der Eroberung der Stadt durch die Türken im Jahr 1521 ansetzen und sich auf die folgenden rund vierzig Jahre erstrecken. Im Defter aus dem Jahr 1528 las der Herausgeber Dolne (Dolnji, 'Unter') und Korne (Gornji, 'Ober') Šemcin, in den drei nachfolgen-

* Филозофски факултет универзитета у Београду, Чика Љубина 18-20, 11000 Београд, Србија; loma.aleksandar@gmail.com

1 Die in LP 1116 angegebenen Belege stammen aus Denkmälern serbischer und russischer Redaktion, vgl. auch Sreznevskij 1517.

2 Im Original Gело чиготово Аоврослава грьвице и сь своими мегтами кегва половина (BH 1: fol. 48r), was sich als „,das Dorf des Tschigots Dobroslav Grbica, mit seiner Gemarkung, eine Hälfte davon (wird dem neugegründeten Kloster geschenkt)“ interpretieren lässt.

3 Vgl. Vasmer 4: 360 (ohne Deutungsvorschlag) und Loma 2013 a. a. O., wo ein Zusammenhang mit mitteltürkisch jigit, tatarisch źigit 'Jüngling; Held, Dschigit' erwogen wird. 
den Deftern (1528/30, 1536 bzw. 1560) Šemşin. ${ }^{4}$ Da durch den arabischen Buchstaben $c$ [క̌] normalerweise serbisch $\check{c}[\mathrm{t} f]$ und $c$ [ts] wiedergegeben werden, ersehen wir, dass in den 1530er-Jahren die Namenformen Šemčin und Šemšin miteinander konkurrierten, sich jedoch bald danach Letztere durchsetzte, aus der das heutige Šepšin durch den Wandel von $m \check{s}$ zu $p \check{s}$, den man auch anderswo belegen kann, ${ }^{5}$ entstanden ist. Das Verhältnis zwischen Šemčin und Šemšin ist zweideutig, da die beiden Formen urkundlich fast gleichzeitig erscheinen. Man kann nicht ausschließen, dass Šemčin eine sekundäre Entwicklung aus Šemšin vom Typ mundartlich pčenica $<$ pšenica 'Weizen' aufweist; von Šemšin ausgehend, ließe sich der ON als Possessivum zum Personennamen (PN) *Sěmbša erklären, der seinerseits als Hypokoristikum zu *Sěmi-slavъ oder *Sěmb-slavъ aufzufassen wäre. ${ }^{6}$ Doch scheint das Gegenteil plausibler, dassnämlich der Erstbeleg die ältere Namenform abbildet, zumal sie später niemals wiederkehrt. Bei $m \check{c}>m \check{s}$ würde es sich um die Vereinfachung der Konsonantengruppe [mtf] zu [mf] handeln, wofür ich — wie übrigens für $m \check{s}>m \check{c}$ - keine unmittelbare Parallele anführen kann, doch lässt sich neben der oben erwähnten Entwicklung $p \check{s}>p \check{c}$ auch deren Umkehrung, $p \check{c}>p \check{s}$, belegen, etwa im kroatischen ON Tepšići versus Tepčići in der Herzegovina, wohl eine patronymische Bildung zum altserb. und altkroat. Titel tepčij(a), vgl. den altserb. Familiennamen Tepsčićb (14.-15. Jh., Bosnien). ${ }^{7}$ Wie man bald sehen wird, kann hier auch eine semantische Parallele zu unserem ON vorliegen. ${ }^{8}$ Denn Šepčin erweckt den Eindruck, als ob es sich dabei um eine Possessivableitung zu einem Titel auf -čij(a) handle,

4 So in der Umschrift des Herausgebers (Šabanović 1964: 99 f., 216, 240, 308, 318, 555 f.). Die zugrunde liegenden arabischen Graphien sind شمجين bzw. شمشين, wie mir Dr. Srđan Katić vom Istorijski institut Beograd bestätigt hat, der so liebenswürdig war, für mich Šabanović' Lesungen nachzuprüfen; sie erwiesen sich als zuverlässig, mit der Ergänzung, dass im Verzeichnis von 1560 das zweite $s$ ohne Diakritikum (,chapeau“) geschrieben sei, folglich könne man auch Šepsin lesen. Drei spätere Verzeichnisse seien von Šabanović nicht herangezogen worden: In jenem von 1572 lese man ebenfalls Šepsin, im nachfolgenden von 1585 wieder Šemšin, und im jüngsten, von 1741, einmal eindeutig Šemsin, während an einer anderen Stelle sowohl eine Lesung mit inlautendem $\sin$ als auch mit schin möglich sei. Ungeachtet dieser orthographischen Schwankungen geht aus der Summe der Belege klar hervor, dass der Name vom 16. bis zum 18. Jh. Šemšin lautete.

5 So wird in einem türkischen Verzeichnis aus dem Jahr 1476 für Westserbien ein Dorf Mšanik (zu *mъšěn ' 'moosig') genannt, das in den etwas jüngeren einheimischen Quellen aus dem 16. bis 17. Jh. als Pšanik begegnet. Heute erscheint der Name zu Šanik vereinfacht (vgl. mundartlich und umgangsprachlich šenica < pšenica 'Weizen'), er bezeichnet einen Teil des Dorfes Bioska westlich von Užice. Gleichen Ursprungs ist wohl der Name einer Ortschaft im benachbarten Gebiet von Dragačevo, der heute ebenfalls Pšanik lautet.

6 So von mir (Loma 2003: 248) vorgeschlagen, vgl. russ. Semšino, ein Dorf in der Oblast Wologda; zum anlautenden $\check{s}$ - s. u.

7 Nach dem RJA (18/1962-1966 s. vv.) geht Tepšići auf einen Spitznamen *Tepša zu tepati 'stammeln' zurück, während für Tepčići neben tepčija auch tepac 'Stotterer' in Betracht gezogen wird, was allerdings beides nicht allzu plausibel erscheint.

8 Angesichts der Tatsache, dass das Dorf Tepšići südlich von Sisak liegt, im selben Teil Kroatiens wie Čigoč, ist vielleicht erwähnensswert, dass auch tepčij, tepčija alttürkischer Herkunft verdächtig ist. 
zumal die prosodische Gestalt der heutigen Namensform Šèpš̄n $<*$ Šepšîn auf eine Kontraktion aus *-ijin hinweist. Ein vergleichbarer Titel ist in der Gründungsurkunde des Klosters Banjska aus dem Jahr 1316 belegt. Im Abschnitt über die Geldstrafen (o globah) liest man dort: sbl'ba sěm'čii 2 dinarja. ${ }^{9}$ Weder sblıba noch sěmbčija kommen sonst in altserbischen Quellen vor. Das erste Wort ist jedoch durchsichtig, es handelt sich um ein Deverbativum auf *-bba von *sblati 'schicken, senden', das im Altrussischen als sol(b)ba 'Abordnung' ein genaues Gegenstück hat (Sreznevskij 3: 742). Damit ist die obige Verordnung als „Vorführung vor den sěmbčija - zwei Dinare“ aufzufassen. In dieser Lautgestalt sonst unbelegt, klingt sěmbčija stark an die Amtsbezeichnung samъčija an und wird mit ihr vom Übersetzer der Urkunde wohl mit Recht gleichgesetzt. ${ }^{10}$ Durch das Hinzuziehen dieser altserbischen Nebenform wird die Frage nach der Etymologie des Wortes sambčija, samčii nur noch verwickelter. ${ }^{11}$ Sonst ist dieses Wort, wie čigoţ, nicht urkundlich, sondern nur literarisch belegt, nämlich in den Übersetzungen des Alten Testaments sowie homiletischer und hagiographischer Schriften. Es steht für diverse Amtsbezeichnungen in verschiedenen Ländern zu verschiedenen Zeiten, im Alten Testament für 'Hausverwalter', ${ }^{12}$ in den Viten für die Bezeichnungen der römischen Reichsämter praefectus ${ }^{13}$ und curator. ${ }^{14}$ Nur aus griechischen und lateinischen Quellen erfahren wir, dass ein entsprechender Titel im Ersten Bulgarischen Reich existierte. Der heilige Kliment von Ochrid wurde nach seiner griechischen Vita in Pliska von

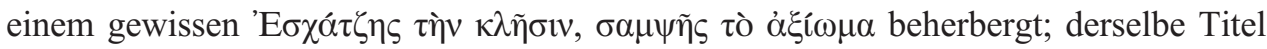
kehrt, in einer verderbten oder abweichenden Form, in der Liste der bulgarischen Gesandten zum Konzil 869-70 in Konstantinopel bei Athanasius Bibliothecarius als camp-

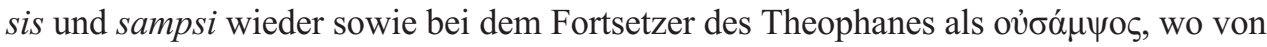

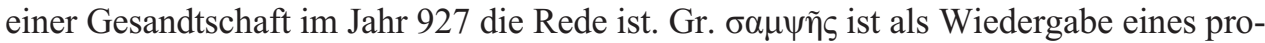
tobulgarischen *samči aufzufassen, mit einem $p$-Einschub in die dem Griechischen fremde Konsonantengruppe. ${ }^{15}$ Vermutlich bezeichnete man damit eine Hofwürde (Run-

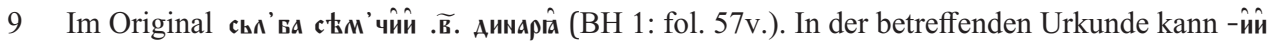
auch für ein $-\bar{\imath}$ stehen, das hier aus einer Dativform auf -ii kontrahiert wäre.

10 Dimitrije Bogdanović, BH 2: 124: „slanje samčiji — 2 dinara“.

11 Da die beiden Erstausgaben der Banjska-Urkunde 1890 erschienen, fand altserb. sěmbčija seinen Weg weder in Daničić' (RKS) noch in Miklosichs Wörterbuch (LP) und blieb merkwürdigerweise auch im RJA unbeachtet, so dass es für die etymologische Diskussion zu altksl. samčii bisher nicht herangezogen wurde.

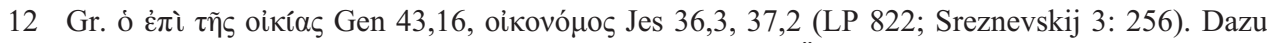
gehört auch der Beleg in der tschechischkirchenslavischen Übersetzung der Homilien des Gregor von Nazianz, wo familiaritatis locum apud divitem meruit der lateinischen Vorlage als $\operatorname{sam}(o)$ čija město u bogatago priimetb wiedergegeben wird (ESJS 793 s. vv. samъčii, sanъ).

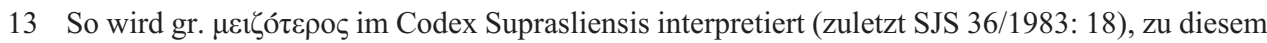

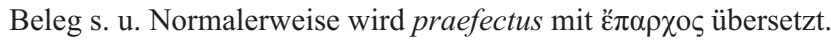

14 In der Vita des heiligen Eutychius, Bischof von Konstantinopel: eparhovъ samčï, gr. $\tau \tilde{\omega} v$

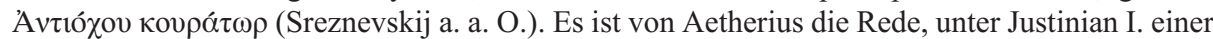
der führenden Senatoren und curator domus divinae Antiochi (PLRE 3: 21).

15 Folglich wäre der Halbvokal der altksl. Form als bloße orthographische Konvention zu beurteilen. 
ciman 1930: 286). Es handelt sich wohl um ein protobulgarisches Wort, dem wahrscheinlich das turksprachige Etymon von altksl. sanъ 'Würde, Rang' zugrunde liegt. ${ }^{16}$ Wenn altserb. sěm'čija hierher gehört, erfordert sein ě eine Erklärung, da samъčii, samъčija sonst stets den Wurzelvokal $a$ aufweist. An eine orthographische Entgleisung ist schon deshalb schwer zu denken, da ein Schwanken zwischen $a$ und $e$ der damaligen Rechtschreibung von Raszien (,raški pravopis“), für die die Banjska Urkunde als beispielhaft gelten darf, fremd ist. Wenn die oben vorgeschlagene Interpretierung des ON Šepšin < Šemčin zutrifft, erweist sich dadurch die altserbische Wortform mit $\check{e}$ in der ersten Silbe als real. Sie kann aus samčij(a) durch die Anlehnung an ein einheimisches Wort entstanden sein, am ehesten an eine Ableitung von *sěmbja 'Familie', wie aruss. sěmьса 'jüngeres, untergeordnetes Familienmitglied, Knecht' (Belehrung des Wladimir Monomach). Das Lehnwort samčij dürfte als Adjektiv dazu vom Typ *dětbčbjb > neuserb. $d(j) e \check{c}(i) j i$ 'kindlich, Kinder' zu dětbca 'Kinder' umgedeutet worden sein: *sěmči $j$ 'für die Dienerschaft (am Hof) zuständig', was durch samčij(a) 'Hausverwalter' nahegelegt worden sein könnte. Die angenommene Umbildung konnte nur zu einem Zeitpunkt stattfinden, als die Wortfamilie ursl. dial. *sěmb 'Person', *sěmbja 'Familie' auf südslavischem Boden noch in Gebrauch stand. Dafür, dass diese Wörter ehemals auch im slavischen Süden und Westen bekannt waren, legt vor allem die Onomastik Zeugnis ab (vgl. ESJS 804). Im altserbischen Raum lässt sich eine Reihe von PN belegen, die den Stamm *sěm(i)- enthalten: *Sémigněvъ, *Sémidorgъ, *Sěmitěxъ, *Sémiradı, *Sémęta, *Sěmixъ, *Sěmъkъ. Sie alle sind jedoch aus ON rekonstruiert (Loma 2003 passim) und in den altserbischen Quellen nicht unmittelbar bezeugt, die für das Onomastikon des 13. bis 15. Jh. recht ergiebig sind, woraus ein Argumentum e silentio hervorgeht, das für die Entstehung der Form *sěmčij > sěmčija den Terminus ante quem liefern kann. Angesichts der gut begründeten Annahme, dass der Teil Serbiens, wo Šepšin liegt, im Mittelalter und in der frühen Türkenzeit zum nordserbischen Mundartbereich gehörte, wo *̌̌ seinen phonemischen Charakter als ein von $e(<* e, *)$ geschiedenes $e$ bewahrt hat, darf man hinter dem Erstbeleg von 1528 eine Lautgestalt Ṣ̌mčîn vermuten, die aus ursprünglichem *Sěmčijin durch reguläre Vokalkontraktion in der zweiten Silbe und Assimilation des anlautenden $s$ wie in šiče < siče 'zischt', šušanj < sušanj 'trockenes Laub', Šušeoka 'Dorf bei Valjevo in Nordwestserbien' < sušelka 'getrocknete Feige' (als Familienname $)^{17}$ entstanden ist. Andererseits weisen die ON Šemihova ${ }^{18}{ }^{5}$ Šmgonj ${ }^{19}$

16 Vgl. ESJS a. a. O., Vasmer 3: 554-555 s. vv. самчuй, сан, Räsänen 400; bemerkenswert ist die

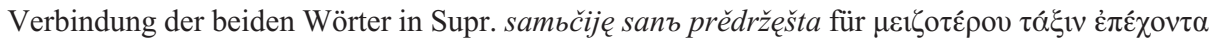

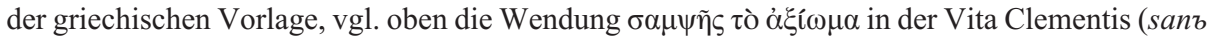
$=\dot{\alpha} \xi \hat{i} \omega \mu \alpha)$.

17 Ab 1528 in denselben Deftern wie Šemčin/Šemšin als Šwšwka belegt, worin sich am ehesten eine Pluralform Šušeoke verbirgt.

18 Ein ab 1475/77 belegtes Dorf in Ostbosnien, unweit von Goražde.

19 Heute Šengolj in Westserbien, zum ersten Mal 1413 in einer Urkunde als Шєм 'rw in den türkischen Verzeichnissen aus dem Zeitraum 1479-1572 kehrt diese Namensform wieder, der *Sěmb-goj-bn-, eine Possessivableitung (zur Bildung s. Loma 2013: 246 f.) vom PN *Sěmbgojb (mit kurzem Bindevokal des $i$-Stammes) zugrunde zu liegen scheint. 
sowie der altserb. Familienname Šemković ${ }^{20}$ auf eine Sonderentwicklung von ursl. *sěmzu šem- hin. Ein anderes Šemšin wird im ausführlichen Steuerregister des Sandschaks Smederevo aus dem Jahr 1585 verzeichnet, als der Fähre von Boğurdelen, heute Šabac an der Save, zugehörig. ${ }^{21}$ Wahrscheinlich gehört hierher auch Šamšin, heute ein Flurname im syrmischen Dorf Berak (Popović 1950: 43) und im türkischen Verzeichnis von 1570 als Dorf belegt, der bezeichnenderweise ein $a$ in der Initialsilbe aufweist. ${ }^{22}$ Das Wiederkehren des Namens in Syrmien lässt sich am einfachsten dadurch erklären, dass er dorthin von Ansiedlern aus Nordserbien übertragen wurde, die zur Zeit der türkischen Eroberungen in Südungarn Zuflucht suchten.

Anknüpfend an die obige Erörterung von Šepšin $<$ Šemčin möchte ich erneut auf einen anderen ON aus derselben Gegend, Vř̌̀n n, eingehen, da er eine Parallelbildung aufzuweisen scheint. Bisher war er unterschiedlich gedeutet worden. Der Umstand, dass in der Nähe von Vrčin eine römische Inschrift gefunden wurde, die eine Widmung an die Göttin Orcia enthält, hat Vladimir Ćorović (1937: 233-234) zu einer Deutung inspiriert, die Vrčin auf *Orcinum, ein lateinisches Adjektiv zu diesem sonst unbekannten Götternamen, zurückführt. Kulturgeschichtlich gesehen war diese Ableitung durchaus plausibel und auch die angenommene Lautentwicklung erschien einwandfrei, ${ }^{23}$ aber nur bis zur 1964 erfolgten Veröffentlichung der obengenannten türkischen Verzeichnisse, wo das heutige Vrčin zwischen 1528 und 1560 fünfmal genannt wird, jeweils als Hrčin. ${ }^{24}$ Durch die Erkenntnis, dass dieser ON ursprünglich mit einem $h$ - anlautete, wurde seine Substratetymologie widerlegt und die Suche nach seiner Herkunft in eine andere Richtung gelenkt. Angesichts eines alttschech. PN Chrtek zu ursl. *xъrtъ 'Windhund', eines altschech. ON Chrčice sowie eines skr. Toponyms Hrtkovci (in Syrmien) nahm ich zuerst eine Possessivableitung auf -inъ von ${ }^{*} X$ brtъka (als femininem Gegenstück zu ${ }^{*} X$ ъrtъ $k ъ$ ) an (Loma 1991: 112), obwohl ein solcher Frauenname unbelegt und überhaupt die Wahrscheinlichkeit nicht allzu hoch ist, dass ein Dorf im Mittelalter nach einer Frau benannt wurde. Nachdem mir jedoch ein anderes Hrčin ins Blickfeld gerückt war, ein Bach, der vom 13. bis zum 15. Jh. im Gebiet von Turopolje als Hurchin, Hercin, Hrechin urkundlich bezeugt ist, verzichtete ich auf meine frühere Deutung dieses Namens als Possessivum und schrieb ihm lieber eine deskriptive Bedeutung zu, nämlich 'Hamsterbach', zu ursl. dial. (serbokroatisch, slovenisch, slovakisch) *xъrčbkъ 'Hamster' (Loma 1997: 17). Diese Etymologie halte ich weiterhin für plausibel. Wenn aber Šepšin < Šemčin wirklich auf altserb. *Sěmčijin zurückgeht, ist für Vrčin < Hrčin auch auf eine dritte Deutungsmöglichkeit hinzuweisen: Als Possessivableitung kann dieser ON von der sonst unbelegten Hofamtsbezeichnung

20 Ein bosnischer Edelmann aus dem späten 14. Jh. (RKS 3: 486).

21 Dr. Srđan Katić, brieflich.

22 McGowan 1983: 450, zweimal, im ersten Beleg mit S- (سامشين), danach aber eindeutig Şamşin geschrieben.

23 Aus lat. tautosyllabischem or- hätte sich erwärtungsgemäß slav. v-br-, mit prothetischem $v$ - im Anlaut, ergeben; die Palatalisierung von lat. $c i$ wird durch das entlehnende Slavische oder - so bei Popović 1960: 103 - durch das vermittelnde Romanische erklärt.

In Transkription Hirçin (Šabanović 1964: 70, 198, 376, 566, 567). 
*xъrtbčii 'für Windhunde zuständig' abgeleitet sein, vgl. hinsichtlich Bildung und Bedeutung alttschech. lovčí, altpoln. łowczy, altruss. lovbčii 'Hofjägermeister'. Die beiden Dörfer Šepšin und Vrčin liegen in Bachtälern, Vrčin an einem gleichnamigen Wasserlauf, während das Gewässer, das das Dorf Šepšin durchfließt, auf der Karte als Ravan 'Ebene' bezeichnet ist, wobei es sich um ein sekundäres Hydronym handeln dürfte. Vermutlich bezogen sich die maskulinen Possessivadjektive *Sěmčijin und *Hrtčijin ursprünglich auf potok 'Bach' oder dol 'Tal', um das Lehen eines sěmčija bzw. eines *hrtčija zu bezeichnen.

Ungeachtet der Tatsache, ob diese Erwägungen zutreffen oder nicht, lässt sich dadurch einmal mehr der Grundsatz bestätigen, dass in der Ortsnamenforschung nur die historischen Belege und nicht die modernen Namenformen einen sicheren Ausgangspunkt für die etymologische Deutung bilden. Es waren ja die türkischen Quellen aus dem 16. Jh., die es uns ermöglichten, die Frage nach der Herkunft der heutigen ON Šepšin und Vrčin seriös zu betrachten und somit vielleicht indirekt einen Blick in die feudalen Verhältnisse früherer Zeiten in der Umgebung von Belgrad zu werfen.

\section{Quellenverzeichnis}

BH = Povelja kralja Milutina manastiru Banjska - Svetostefanska hrisovulja. I-II. Angepasst von Đ. Trifunović. Beograd: Muzej u Prištini, Mnemosyne - Centar za očuvanje nasleđa Kosova i Metohije, Službeni glasnik, 2011. [Kyrillisch.]

DANIČIĆ, Đuro (1863-1864) Rječnik iz književnih starina srpskih. Biograd: Državna štamparija. [Kyr.]

KARADŽIĆ, Vuk Stefanović (1827) „Geografičeskostatističesko opisanije Srbije.“ Danica. Zabavnik za godinu 1827, 25-120. [Kyr.]

\section{LP s. MIKLOSICH}

McGOWAN, Bruce W. (1983) Strem sancaği mufassal tahrır defterı. Ankara: Türk Tarih Kurumu Yayınları.

MIKLOSICH, Franz (1862-1865) Lexicon Palaeoslovenicograecolatinum. Vindobonae: Guilelmus Braumueller.

RJA = Rječnik hrvatskoga ili srpskoga jezika. I-XIX. Zagreb: Jugoslavenska akademija znanosti i umjetnosti, 1880-1967.

RKS s. DANIČIĆ.

SJS = Slovnik jazyka staroslověnského. I-LII. Praha: Slovanský ústav Československé akademie věd, 1958-1997.

SREZNEVSKIJ Vjačeslav Izmailovič (1893-1903) Materialy dlja slovarja drevnerusskago jazyka. I-III. SanktPeterburg: Tipografija Imperatorskoj akademii nauk. [Kyr.]

ŠABANOVIĆ, Hazim (1964) Turski izvori za istoriju Beograda. I/1: Katastarski popisi Beograda i okoline 1476-1566. Beograd: Istorijski arhiv Beograda. [Kyr.] VUK s. KARADŽIĆ 


\section{Literaturverzeichnis}

ĆOROVIĆ, Vladimir (1937) „Otkud ime Vrčin“. In: Zbornik lingvističkih i filoloških rasprava A. Beliću o četrdesetogodišnjici njegova naučnog rada. Beograd: Mlada Srbija, 233-234 [Kyr.].

ESJS = Etymologický slovník jazyka staroslověnského. Praha: Ústav pro jazyk český Akademie věd České republiky/Academia, $1989 \mathrm{ff}$.

FASMER, Maks, Étimologičeskij slovar' russkogo jazyka. I-IV. Übersetzt und ergänzt von O. N. Trubačeva. Moskva: Progress, 1986-1987. [Kyr.]

LOMA, Aleksandar (1991) „Vorslavisches Substrat in der Toponymie Serbiens. Bisherige Ergebnisse, Probleme und Perspektiven weiterer Erforschung“. Die Welt der Slaven 36 (N. F. 15)/1-2, 99-139.

Ders. (1997) ,Srpskohrvatska geografska imena na ina, mn. ine: pregled tipova i problemi klasifikacije“. Onomatološki prilozi 13, 1-26. [Kyr.]

Ders. (2002-2003) „Jedna srpskoukrajinska toponomastička paralela - Semegnjevo: Semygyniv." Naš jezik 34/3-4, 244-251. [Kyr.]

Ders. (2013) Toponimija Banjske hrisovulje. Beograd: Srpska akademija nauka i umetnosti. (Biblioteka Onomatoloških priloga, knj. 2.) [Kyr.]

PLRE $=$ Prosopography of the Later Roman Empire. I-III. Cambridge: Cambridge University Press, 1971-1992.

POPOVIĆ, Dušan J. (1950) Srbi u Sremu do 1736/7. Beograd: Srpska akademija nauka. (Posebna izdanja CLVIII, Etnografski institut, 1.) [Kyr.]

POPOVIĆ, Ivan (1960) „Bemerkungen über die vorslavischen Ortsnamen in Serbien.“ Zeitschrift für slavische Philologie 28, 101-114.

RÄSÄNEN, Marti (1969) Versuch eines etymologischen Wörterbuchs der Türksprachen. Helsinki: Suomalais-Ugrilainen Seura.

RUNCIMAN, Steven (1930) The First Bulgarian Empire. London: G. Bell \& Sons.

VASMER s. FASMER

\section{Resümee \\ ZU EINIGEN MITTELALTERLICHEN AMTSBEZEICHUNGEN IN DER TOPONYMIE SERBIENS}

Die vorliegende Arbeit behandelt den Ortsnamen Šepšin, wie ein Dorf in Serbien unweit von Mladenovac, südlich von Belgrad, heißt. Nach dem Zeugnis der türkischen Verzeichnisse des 16. Jh.s, hat sich die heutige Form des Namens über Šemšin aus Šemčin entwickelt, wie sein Erstbeleg aus 1528 lautet. Vorausgesetzt, dass sich das anlautende $\check{S}$ - aus $S$ - durch Assimilation vom Typ šiče < siče 'zischt' entwickelt hat, lässt sich die ursprügliche Namenform als *Sěmčijin wiederherstellen, wo sich um ein Possessivadjektiv zur altserbischen Amtsbezeichnung sěmbčija handelt. Nur als Hapax in der BanjskaUrkunde aus 1316. belegt, wird sie mit aksl. samъčii 'praefectus' gleichgesetzt, wo wahrscheinlich ein Lehnwort aus dem Protobulgarischen vorliegt. In die- 
sem Fall liesse sich das $\check{e}$ der altserbischen Nebenform durch den Einfluss des urslav. Wortes *sěmb 'Familie' erklären. Anknüpfend wird die Frage nach der Herkunft eines anderen Ortsnamens aus demselben Gebiet, Hrčin, heute Vrčin, wieder in Betracht gezogen. Bisher unterschiedlich gedeutet, lässt sich er analogisch zu *Sěmčijin auf *Hrtčijin als Possessivbildung zur sonst unbelegten Hofamtsbezeichnung *xъrtbčii 'für Windhunde ( *xъrtъ) zuständig, Hofjägermeister' zurückführen. In beiden Fällen dürfte sich das maskuline Possessivadjektiv auf -in ursprünglich auf potok 'Bach' oder $d o l$ 'Tal' im Lehensbesitz der betreffenden Person bezogen haben.

Schlüsselwörter: Ortsnamenkunde, Serbien, Mittelalter, Amtsbezeichnungen

\section{Abstract \\ SOME MEDIEVAL TITLES IN THE TOPONYMY OF SERBIA}

The paper deals with the place name Šepšin, designating a village in Serbia situated to the south of Belgrade, near the town of Mladenovac. According to the testimony of the Turkish $16^{\text {th }}$ century tax registers, its modern form developed via Šemšin from the first recorded Šemčin (1528). On the assumption that the initial $\breve{S}$ - derives from $S$ through the assimilation of the type šiče < siče 'hisses', the original form of the name may be reconstructed as *Sěmčijin, a possessive adjective derived from Old Serbian title sěmbčija. The underlying term, a hapax attested in the Banjska charter from 1316, is assumed to be the same as Old Church Slavic samъciii 'prefect', which is probably a loanword from Proto-Bulgarian. In that case, the $e$ of the Old Serbian variant may be due to the influence of Common Slavic *sémb 'family'. In addition, another place name from the same region is reconsidered, Hrčin, today Vrčin, which displays a similar derivation. It has been variously interpreted so far, but by analogy with *Sěmčijin a proto-form *Hrtčijin seems rather probable, deriving from the unattested title *xъrtbčii, which presumably designated an official at court charged with greyhounds $(x b r t i)$ and generally with hunting. In both cases the masculine adjective in -in may have originally referred to potok 'creek' or dol 'valley' as a part of the feudal estate held by the official in question.

Keywords: toponymy, Serbia, Middle Ages, titles

\section{Povzetek \\ O NEKATERIH SREDNJEVEŠKIH URADNIŠKIH NAZIVIH V TOPONIMIJI SRBIJE}

V prispevku se obravnava toponim Šepšin, kot se imenuje vas v Srbiji blizu Mladenovca, južno od Beograda. Glede na pričevanje turških popisov XVI. stoletja je sodobna oblika imena nastala prek oblike Šemšin iz Šemčin, kot se glasi v najstarejšem zapisu iz leta 1528. Ob predpostavki, da je vzglasni $\breve{S}$ - nastal iz $* S$ - z asimilacijo tipa šiče $<$ siče, je 
podana rekonstrukcija prvotne oblike *Sěmčijin, kar bi bil svojilni pridevnik, izpeljan iz starosrbskega naslova sěmbčija. Ta termin je hapaks, izpričan v Banjski hrisovulji leta 1316, in se istoveti s starocerkvenoslovanskim samъčii 'praefectus', verjetno izposojenim iz protobolgarščine. V tem primeru se $\check{e}$ starosrbske variante lahko razloži z vplivom praslovanske besede *sěmb 'družina'. Dodatno se preizkuša etimologija nekega drugega toponima z istega območja, Hrčin, danes Vrčin, ki izkazuje podobno tvorjenost. Doslej so ga različno razlagali, toda po analogiji s *Sěmčijin je mogoče s precejšnjo verjetnostjo predpostaviti prvotno *Hrtčijin, izpeljano iz neizpričanega *xъrtbčii kot poimenovanja za dvornega uradnika, zadolženega za hrte in za lov sploh. $\mathrm{V}$ obeh primerih se je pridevnik na -in $\mathrm{v}$ moškem spolu prvotno nanašal na potok ali dol kot del fevdalne posesti $v$ lasti omenjene osebe.

Ključne besede: toponimija, Srbija, srednji vek, uradniški nazivi 